\title{
Editorial
}

\section{Qualitative Analysis of Dynamic Activity Patterns in Neural Networks}

\author{
Ivanka Stamova, ${ }^{1}$ Haydar Akca, ${ }^{2}$ and Gani Stamov ${ }^{3}$ \\ ${ }^{1}$ Center of Computer Science and Engineering, Burgas Free University, 8000 Burgas, Bulgaria \\ ${ }^{2}$ Department of Applied Sciences and Mathematics, College of Arts and Sciences, Abu Dhabi University, \\ P.O. Box 59911 Abu Dhabi, UAE \\ ${ }^{3}$ Department of Mathematics, Technical University of Sofia, 8800 Sliven, Bulgaria \\ Correspondence should be addressed to Ivanka Stamova, stamova@bfu.bg
}

Received 3 May 2011; Accepted 3 May 2011

Copyright (C) 2011 Ivanka Stamova et al. This is an open access article distributed under the Creative Commons Attribution License, which permits unrestricted use, distribution, and reproduction in any medium, provided the original work is properly cited.

Neural networks have recently been widely used to model some of the human activities in many areas of science and engineering. Mathematical modeling in neural networks has been based on "neurons" that is different both from real biological neurons and from the realistic functioning of simple electronic circuits. These models have received increasing interest due to their impressive applications in areas such as classification, parallel computing, associative memory, pattern recognition, computer vision, and solving some optimization problem. Such applications heavily depend on the dynamic behavior of networks; therefore, the qualitative analysis of these dynamic behaviors is a necessary step for practical design of neural networks.

Neural networks have broad applicability to real-world business problems. In fact, they have already been successfully applied in many industries. Since neural networks are best at identifying patterns or trends in data, they are well suited for prediction or forecasting needs including sales forecasting, industrial process control, customer research, data validation, risk management, and target marketing.

In this special issue on multimedia networking, we have invited a few papers that address such issues.

In the first paper, shunting inhibitory cellular neural networks with delay are studied. By using the Lyapunov functional and contraction mapping, a set of criteria are established for the global exponential stability, the existence, and uniqueness of pseudo-almost-periodic solutions.

The second paper is on a bidirectional associative memory (BAM) model. Several interesting properties of the BAM architecture have been investigated. The analysis of the 
transmission function, as well as one-to-one association and many-to-one association are studied. It was shown that simple time-delay Hebbian learning can perform one-to-one association and many-to-one association with both binary and real-valued pattern. More complex architectures are explored by using the multidirectional associative memory (MAM) architecture while keeping the same learning and transmission functions. Simulations show the BAM's various capacities by using several types of encoding and recall situations.

The third paper describes a novel hysteresis identification method based on numerical classical Preisach model by use of artificial neural networks. The experimental data showed that the new approach provides accurate hysteresis nonlinearity modeling in comparison with the classical Preisach model and can be used for many applications such as hysteresis nonlinearity control and identification in SMA and Piezo actuators and performance evaluation in some physical systems such as magnetic materials.

The fourth paper presents an adaptive neuro fuzzy interface system approach to identify the real power transfer between generators. The proposed method could be adapted to true application of real power allocation and help to resolve some of the difficult real power pricing and costing issues to ensure fairness and transparency in the deregulated environment of power system operation.

Finally, the fifth paper of this special issue is devoted to an empirical-statistical downscaling method for precipitation prediction which uses a feed-forward multilayer perceptron neural network. The downscaling model has taken into account the physical bases of the precipitation evolution induced by meteorological and land-surface characteristics in the study area. As a result, the present model has exhibited cost-effective, simple-toimplement, and universal application.

Ivanka Stamova Haydar Akca Gani Stamov 


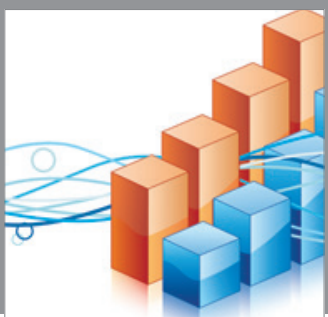

Advances in

Operations Research

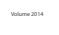

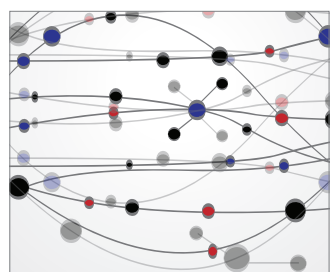

\section{The Scientific} World Journal
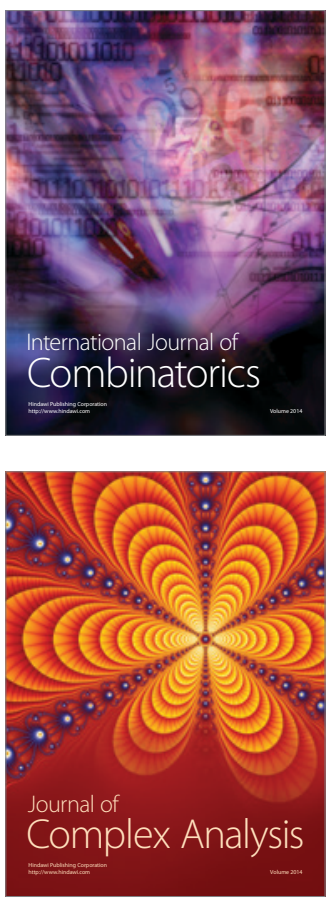

International Journal of

Mathematics and

Mathematical

Sciences
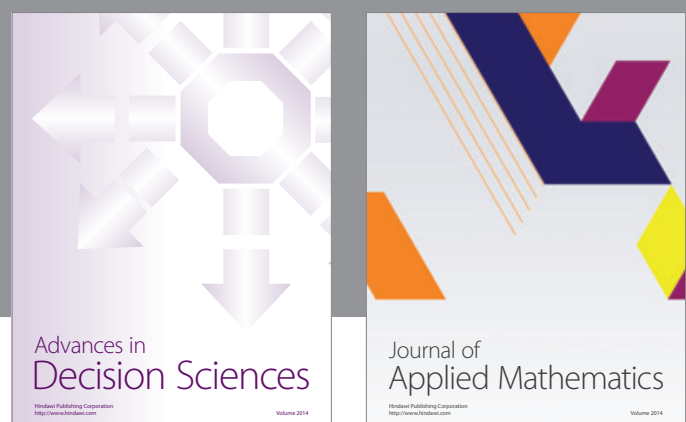

Journal of

Applied Mathematics
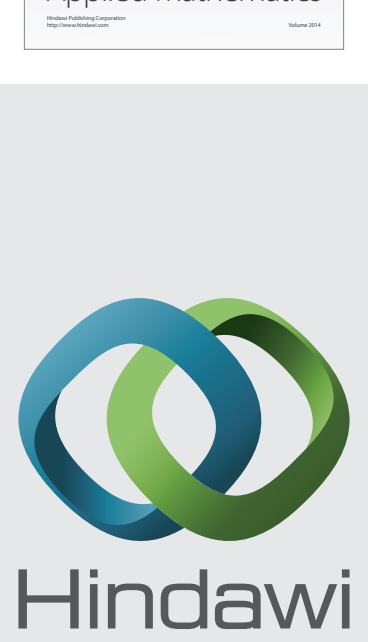

Submit your manuscripts at http://www.hindawi.com
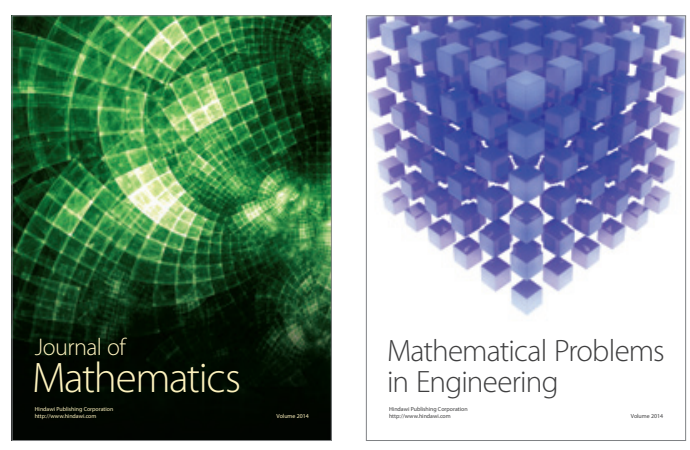

Mathematical Problems in Engineering
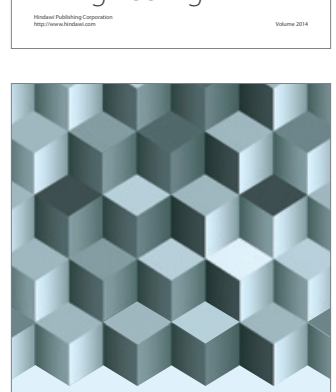

Journal of

Function Spaces
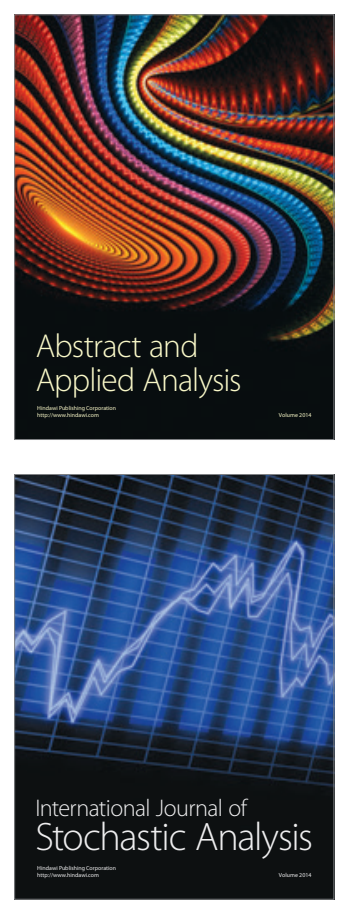

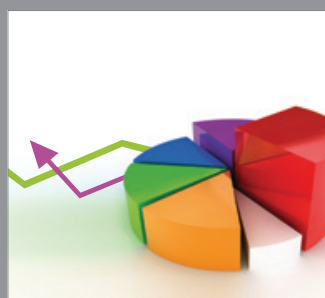

ournal of

Probability and Statistics

Promensencen
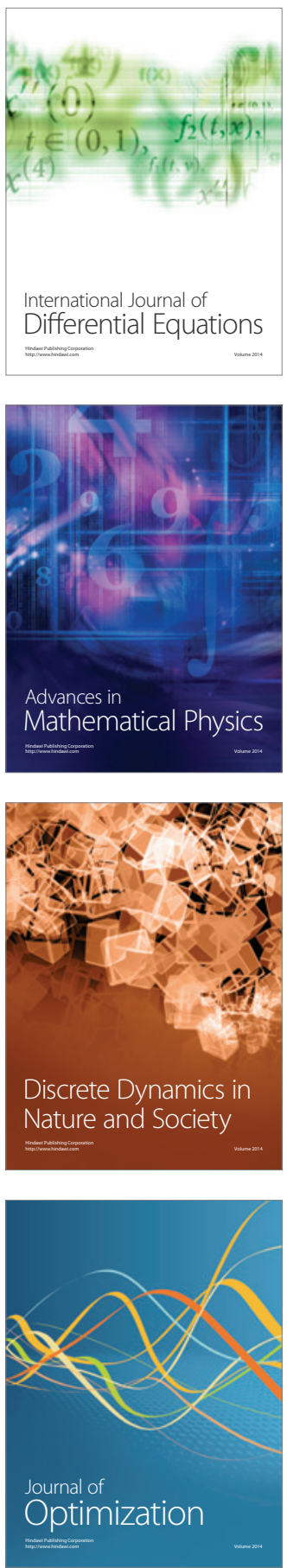